\title{
PAX2 is an antiapoptotic molecule with deregulated expression in medulloblastoma
}

\author{
M.C. BURGER ${ }^{1 *}$, D.P. BRUCKER ${ }^{1 *}$, P. BAUMGARTEN ${ }^{2}$, M.W. RONELLENFITSCH ${ }^{1}$, C. WANKA $^{1}$, \\ M. HASSELBLATT ${ }^{3}$, M.R.ECCLES ${ }^{4}$, T. KLINGEBIEL ${ }^{5}$, M. WELLER $^{6}$, \\ J. RIEGER ${ }^{1}$, M. MITTELBRONN ${ }^{2}$ and J.P. STEINBACH ${ }^{1}$
}

${ }^{1}$ Dr Senckenberg Institute of Neurooncology, Goethe-University Hospital Frankfurt, Frankfurt; ${ }^{2}$ Institute of Neurology

(Edinger Institute), Goethe-University Hospital Frankfurt, Frankfurt; ${ }^{3}$ Institute of Neuropathology, University Hospital

Münster, Münster, Germany; ${ }^{4}$ Department of Pathology, Dunedin School of Medicine, University of Otago,

Dunedin, New Zealand; ${ }^{5}$ Klinik für Kinder- und Jugendmedizin III, Goethe-University Hospital Frankfurt,

Frankfurt, Germany; ${ }^{6}$ Department of Neurology, University Hospital Zürich, Zürich, Switzerland

Received November 30, 2011; Accepted February 6, 2012

DOI: 10.3892/ijo.2012.1446

\begin{abstract}
P A X 2$ is a paired box transcription factor possessing a fundamental role in the embryogenesis of hindbrain and urinary tract. $P A X$ genes are proto-oncogenes, $P A X 2$ expression may contribute to the pathogenesis of renal cell carcinoma. Because of the expression of $P A X 2$ in the developing hindbrain and its essential role in cerebellar development, it has been hypothesized that $P A X 2$ may also be involved in medulloblastoma tumorigenesis. We investigated the expression pattern of $P A X 2$ and various genes of the neuronal lineage in medulloblastoma and glioma cell lines. We found high expression of PAX2 mRNA and PAX2 protein in medulloblastoma cells and some glioma cell lines independent of their neuronal lineage gene expression signature. Gene suppression of $P A X 2$ decreased the expression of the $P A X 2$ transcriptional target GDNF in Daoy cells and had a profound cytotoxic effect in vitro on Daoy medulloblastoma and T98G glioma cells. Expression of PAX2 was then assessed in two separate medulloblastoma tissue microarrays with a total of 61 patient samples by immunohistochemistry. $P A X 2$ expres-
\end{abstract}

Correspondence to: Michael C. Burger, Dr Senckenberg Institute of Neurooncology, Goethe-University Hospital Frankfurt, Schleusenweg 2-16, D-60528 Frankfurt, Germany

E-mail: michael.burger@kgu.de

*Contributed equally

Abbreviations: DLX1, distal-less homeobox 1; DNA, deoxyribonucleic acid; EMX2, empty spiracles homeobox 2; GFAP, glial fibrillary acidic protein; hASH1, human achaete-scute homolog 1; Ig, immunoglobulin; IHC, immunohistochemistry; MAP2, microtubuleassociated protein 2; mRNA, messenger ribonucleic acid; NGN2, neurogenin-2; NSE, neuron-specific enolase; OLIG2, oligodendrocyte lineage transcription factor 2; PAX, paired box; PBS, phosphate buffered saline; PI, propidium iodide; qPCR, quantitative polymerase chain reaction; TMA, tissue microarray

Key words: PAX2, GDNF, medulloblastoma, glioma, Daoy, T98G sion was detected in the majority of medulloblastoma samples and correlated with less differentiated histology. Therefore, $P A X 2$ is a biomarker for a more aggressive medulloblastoma phenotype and may represent a novel therapeutic target.

\section{Introduction}

The paired box transcription factor gene family has been well characterized both in human $(P A X)$ and mouse (Pax) and consists of nine genes in the human genome which encode the corresponding transcription factors (PAX/Pax). $P A X$ genes, like other developmental control genes, are proto-oncogenes (1). They serve to orchestrate the expression of target gene responses that enable proliferation and suppress apoptosis in their target tissues (2). The expression patterns of $P A X$ genes are therefore tightly regulated both in a spatial and temporal manner during embryonal development. $P A X 2$, in particular, is important for hindbrain and renal development. Interestingly, in hindbrain development, the closely related paralogous $P A X$ genes 2,5 and 8 , can compensate for the loss of one another and a major cerebellar phenotype with partial or complete cerebellar agenesia occurs when two alleles or more are lost (3). In general, $P A X$ genes are downregulated in differentiated tissues. In particular, expression of $P A X 2,5$ and 8 is suppressed in postnatal hindbrain (4).

In line with their biological functions, deregulated overexpression of $P A X$ genes can drive the development of tumors both in children and adults. Specifically, fusion genes involving $P A X 3$ or $P A X 7$ cause alveolar rhabdomyosarcoma (5). An oncogenic fusion gene involving the $\operatorname{Ig}$ enhancer and $P A X 5$ has been found in malignant B-cell lymphomas (6). $P A X 8$ can also be involved in a translocation which results in the PAX8-PPAR $\gamma$ fusion protein in follicular thyroid carcinoma (7). Elevated $P A X 2$ expression has been found in cystic and hyperproliferative disorders of the kidney, possible predecessors of renal cell carcinoma $(4,8,9)$. A high proportion of primary renal cell carcinomas express $P A X 2$ (10). An elevated expression of $P A X 2$ mitigates cisplatin-induced cytotoxicity and blocks caspase- 2 induced apoptosis in ACHN and CAKI-1 renal carcinoma and human embryonic kidney 293 
(HEK293) cells $(11,12)$. Accordingly, inhibition of $P A X 2$ with specific siRNAs in the cell lines EJ (bladder carcinoma) and IGROV-1 (ovarian carcinoma) increased the rate of apoptosis thereby lowering the proliferation rate (13).

Due to the expression pattern of $P A X 2,5$ and 8, particular interest has been generated in these genes as candidate genes involved in medulloblastoma, the most common malignant embryonal brain tumor that originates from the hindbrain. We therefore investigated the expression pattern of $P A X 2$ and $P A X 8$ in addition to PAX5 in medulloblastoma cells and compared it to their expression in glioma cells, in which expression of $P A X 5$ has been shown to correlate inversely with the expression of p53 (14). We found high expression of $P A X 2$ in medulloblastoma cells and the majority of medulloblastoma samples from two separate tissue microarrays. Gene suppression of PAX2 had a profound cytotoxic effect in vitro.

\section{Materials and methods}

Cell culture and reagents. Daoy medulloblastoma cells were a gift from T. Pietsch (Bonn, Germany). The human malignant glioma cell lines were kindly provided by N. De Tribolet (Lausanne, Switzerland). The murine glioma cell line SMA-560 was a gift of D. Bigner (Durham, NC, USA). The immortalized astrocytic cell line SV-FHAS was kindly provided by A. Muruganandam (Ottawa, Canada). EJ bladder carcinoma cells were a gift from M. Eccles (Otago, New Zealand). Cells were cultivated as described before (15). Cell lines were stably transfected by lipofection using FuGENE6 (Roche, Mannheim, Germany). The plasmids encoding human $P A X 2$ transcription variants $P A X 2 b$ or $P A X 2 c$ (pCMV/PAX2-N1 and $\mathrm{pCMV} / P A X 2$ $\mathrm{N} 3$, respectively, plus the empty control vector $\mathrm{pHN} / \mathrm{CMV}$ ) were kindly provided by $\mathrm{M}$. Eccles. The plasmid encoding murine Pax2 (pmPax2/334 plus the empty vector pKW10) was kindly provided by M. Busslinger (Vienna, Austria).

Cell growth and hypoxia was assessed by crystal violet staining as previously described (15). Cell viability was analysed by lactate dehydrogenase (LDH) release assays or flow cytometry (15).

RNA interference. The following small interfering RNA sequences targeting the human $P A X 2$ coding region were used: d1: 5'-AAACUGUCCACACCACUCUUU-3'; d2: 5'-UCUGA UUUGAUGUGCUCUGUU-3'. A predesigned scrambled siRNA was used as control (Dharmacon). For transient siRNA transfections Metafectene Pro was employed according to the protocol with a final siRNA concentration of $50 \mathrm{nM}$ and $0.33 \mu \mathrm{l}$ Metafectene Pro in $100 \mu \mathrm{l}$ transfection medium per well for 96-well plates.

Real-time PCR. RNA was isolated using the RNeasy Mini Kit (Qiagen). Superscript II RT (Gibco) was employed for cDNA synthesis. cDNA amplification was monitored using SYBR Green chemistry (ABgene) on the ABI PRISM 7000 Sequence Detection System (Applied Biosystems). The conditions for all PCR reactions were as follows: 40 cycles of $95^{\circ} \mathrm{C}$ for $15 \mathrm{sec}$ and $60^{\circ} \mathrm{C}$ for $1 \mathrm{~min}$, using the following specific primers (forward and reverse): 18S: 5'-CGGCTACCACATCCAAGG AA-3', 5'-GCTGGAATTACCGCGGCT-3'; DLX1: 5'-TGG AATCCGAACTCCTCATC-3', 5'-TCACATCAGTTGAGG
CTGCT-3'; EMX2: 5'-AGCTTCTAAGGCTGGAACACGC -3', 5'-TACCTGAGTTTCCGTCAGGCTG-3'; hASH1: 5'-AGG AGCTTCTCGACTTCACCAA-3', 5'-ATCACCCTGCTT CCAAAGTCC-3'; NGN2: 5'-AGAGCCAACTAAGATGTTC GTCAAA-3', 5'-CCGATCCGAGCAGCACTAAC-3'; OLIG2: 5'-AACTCCTCCACGTGCTTCCTG-3', 5'-CGGTGCGATCA GAGGAAGG-3'; PAX2: 5'-TGTGGACAGTTTGCGGAAG CA-3', 5'-TGATGTGCTCTGATGCCTGGAA-3'; PAX5: 5'-AAGCGCAAGAGAGACGAAGGT-3', 5'-TGAACAAGTC TCCCCGCATC-3'; PAX6: 5'-GACTTCGGTGCCAGGG-3', 5'-TGGTATTCTCTCCCCCTCCTT-3'; PAX8: 5'-ACAAACG CCAGAACCCTACCAT-3', 5'-TTAATGGAGCTGACAC TGGGCA-3'. Relative mRNA levels were calculated by $2[-\Delta \Delta C(T)]$ method (16).

Immunoblot analysis. Immunoblots were performed as described before (15). Membranes were probed with antiPAX2 antibodies (cat. no. 71-6000, Invitrogen) or anti- $\beta$-actin antibodies (SC-1616, Santa Cruz Biotechnology).

Immunohistochemistry. Immunohistochemistry was performed as described before on paraffin-embedded specimens or cytopellets (17). The polyclonal rabbit anti-human PAX2 (Invitrogen) antibody was used. We investigated two independent tissue microarrays containing medulloblastoma specimens of 61 patients and 3 normal appearing cerebellar specimens from the tumor banks of the departments of neuropathology of the University of Frankfurt ( $n=18)$ and Münster ( $n=43)$. All tissues were evaluated by at least two neuropathologists according to the current classification of tumours of the central nervous system (18) using routine HE, reticulin and immunohistochemical stainings including antibodies against Ki-67, GFAP, MAP2, NeuN, synaptophysin and vimentin. The project was approved by the ethics committee of the university hospital of Frankfurt (project no. 4/09).

TMA evaluation. Immunostained sections were evaluated by two examiners (P. Baumgarten and M. Mittelbronn). A semiquantitative score including staining intensity and frequency similar to commonly used scores in routine pathology was applied (19). The frequency score ranged from 0-4 (score 0 , $0-1 \%$; score $1,1-10 \%$; score $2,11-25 \%$; score $3,26-50 \%$ and score $4,>50 \%$ of all tumor cells showing a positive staining). The intensity score ranged from $0-3$ ( 0 , no staining; score 1 , weak staining; score 2 , moderate staining and score 3 , strong staining). The two results for staining intensity and frequency were multiplied, so that a 'Multi-score' reflected both.

Statistical analysis. Where indicated, a two-tailed Student's t-test was used with $\mathrm{P}<0.05$ considered significant and $\mathrm{P}<0.01$ considered highly significant. Evaluation of survival patterns in mice bearing intracerebral gliomas was performed by the KaplanMeier method. Contingency analysis for PAX2 expression scores derived from the TMA studies according to histomorphological subtypes was performed followed by Pearsons $\chi^{2}$ test.

\section{Results}

PAX2 is highly expressed in Daoy human medulloblastoma cells and some malignant human glioma cell lines. Due to 
A

PAX2
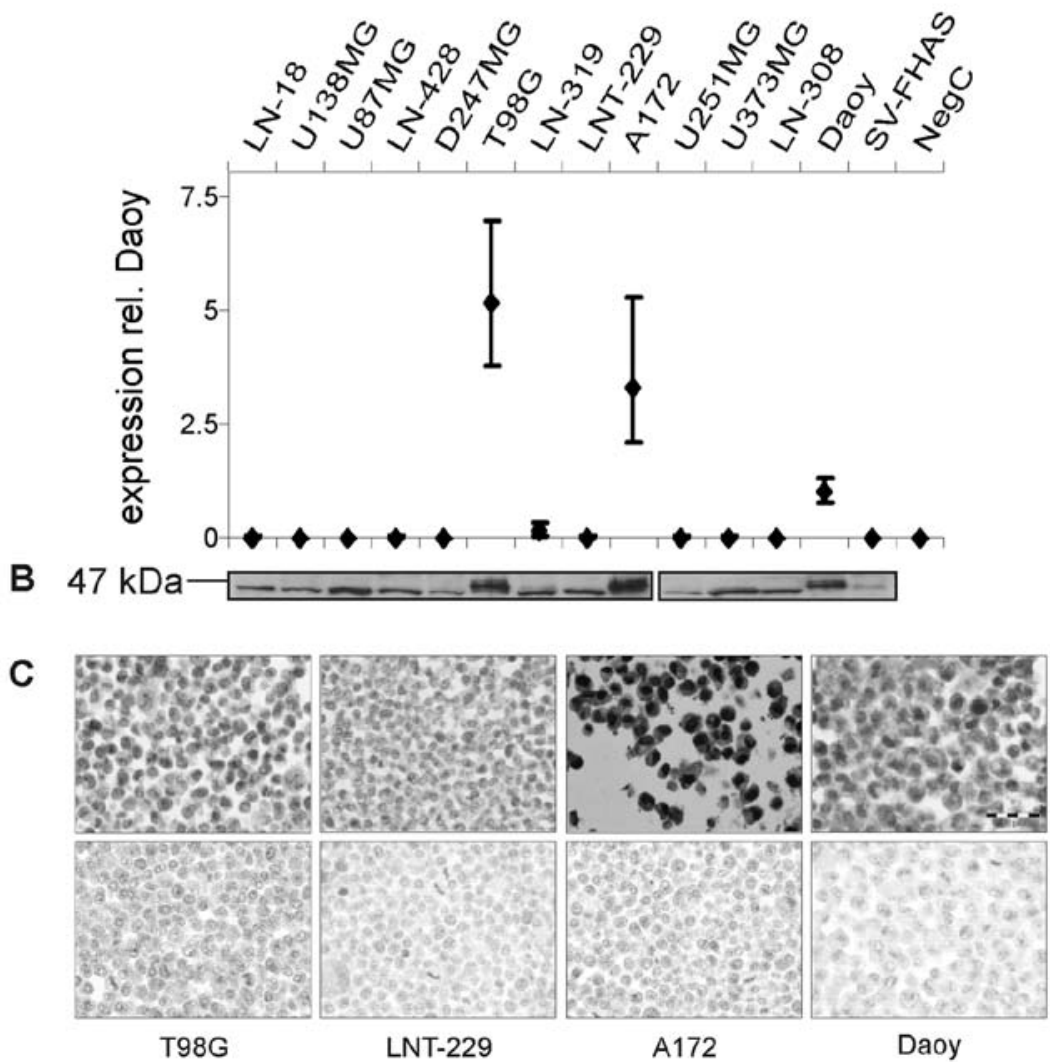

Figure 1.PAX2 is highly expressed in Daoy human medulloblastoma cells and some malignant human glioma cell lines. (A) Daoy medulloblastoma cells and a panel of 12 glioma cell lines plus SV-FHAS were investigated for expression of PAX2 mRNA by RT-quantitative PCR, normalized on the expression level in Daoy. (B) PAX2 expression on the protein level was confirmed by Western blotting. (C) Immunohistochemistry of cell lines T98G, LNT-229, A172 and Daoy (PAX2 upper row, IgG isotype control lower row).

A

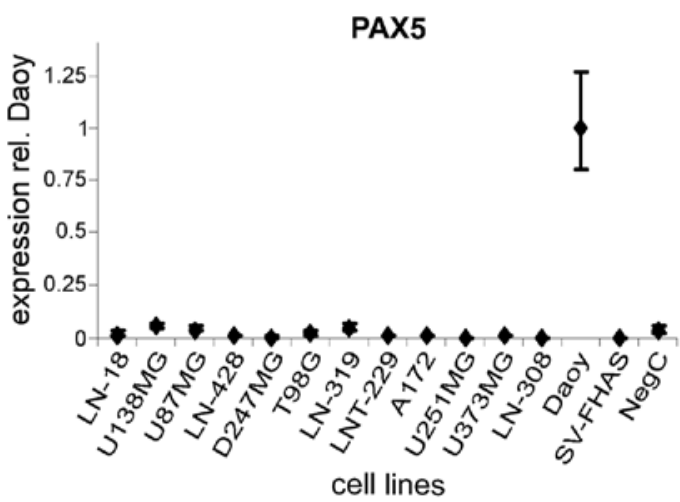

B

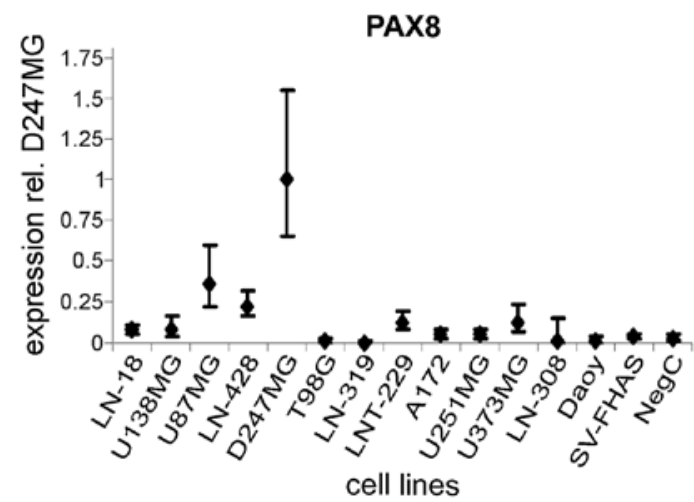

the partial functional redundancy within a PAX subgroup we decided to investigate the expression patterns of $P A X 2$ as well as $P A X 5$ and $P A X 8$. We first screened the human medulloblastoma cell line Daoy and a panel of human malignant glioma cell lines by qPCR. High PAX2 mRNA levels were found in Daoy cells as well as in the glioma cell lines T98G and A172 (Fig. 1A). These results were confirmed by Western blot analysis and immunohistochemistry of cytopellets (Fig. 1B and C). While an unspecific 44-kDa signal was seen in all cell lines tested, the PAX2 specific 47-kDa signal only appeared in cell lines expressing $P A X 2$ in accordance with qPCR results. The origin of the 44-kDa signal seems to be unspecific, as its intensity did not increase in cells transfected with $P A X 2$ (data not shown). In contrast, $P A X 5$ mRNA expression was restricted to Daoy cells (Fig. 2A). PAX8 was not detectable in Daoy cells, but five out of twelve glioblastoma cell lines showed $P A X 8$ mRNA expression (Fig. 2B). We also investigated the mRNA expression of PAX6, whose expression in normal human brain, gliomas and medulloblastomas and confirmed expression of PAX6 in Daoy cells, all

Figure 2. Expression level of $P A X 5$ and $P A X 8$ measured by RT-quantitative PCR. Daoy medulloblastoma cells and a panel of 12 glioma cell lines plus SV-FHAS were investigated for expression of PAX5 (A) and PAX8 (B) mRNA by RT-quantitative PCR. Results were normalized on the expression levels of $P A X 5$ in Daoy and PAX8 in D247MG, respectively. 


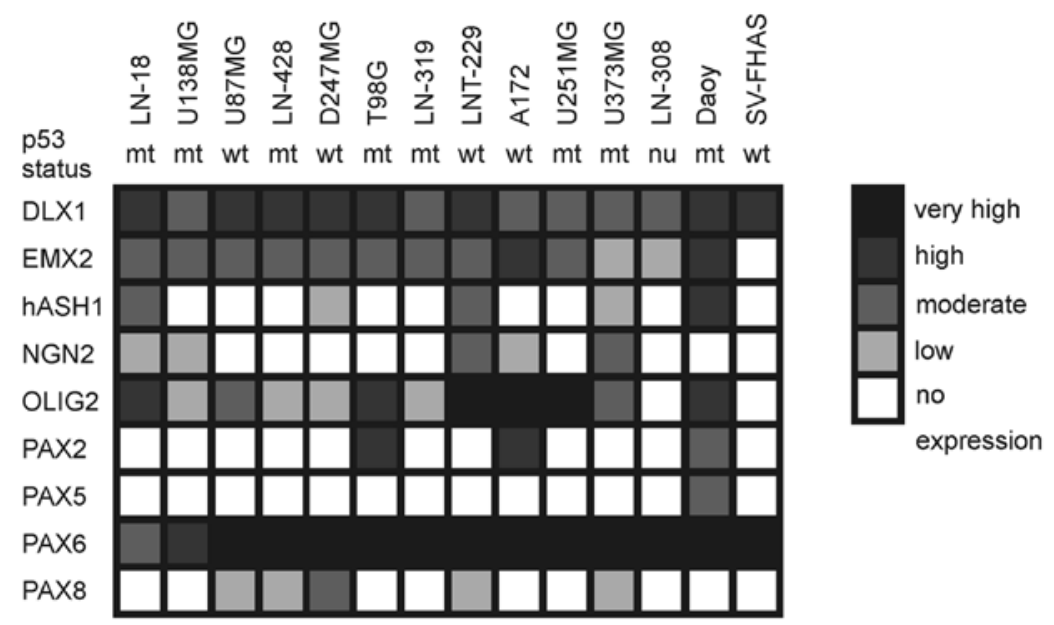

Figure 3. Homeobox and helix-loop-helix transcription factors in glioblastoma and medulloblastoma. Expression pattern of homeobox or helix-loop-helix transcription factors DLX1, EMX2, hASH1, NGN2 and OLIG2 compared to the expression of PAX2, PAX5, PAX6 and PAX8. The amount of expression on mRNA level was measured by qPCR and evaluated according to ref. 13. No correlation between the expression of PAX transcription factors and homeobox or helix-loophelix transcription factors were observed. No correlation with p53 status existed according to ref. 25 (wt, wild-type; mt, mutant; nu, null).

A

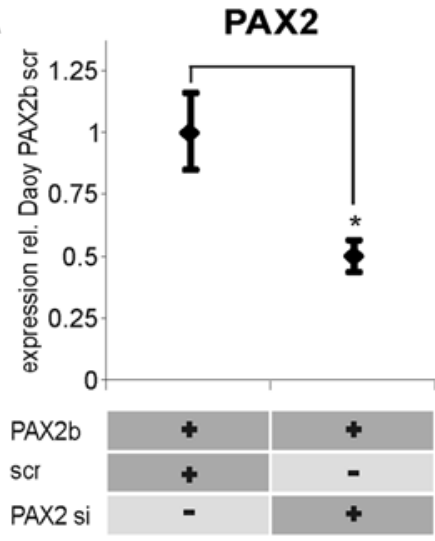

B

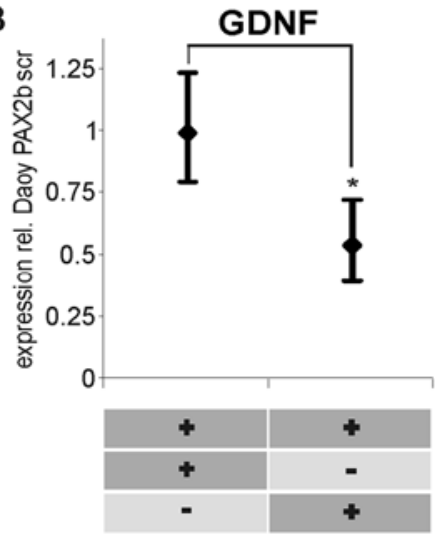

C

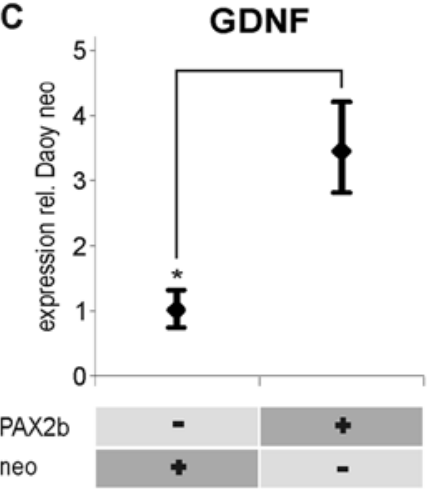

D

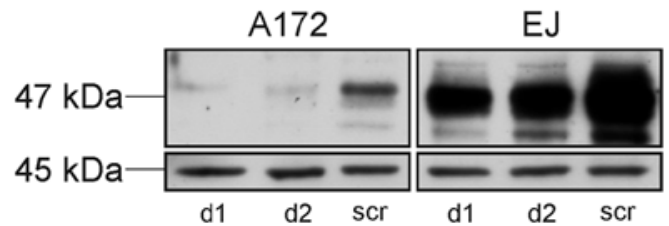

Figure 4. GDNF is regulated by $P A X 2$ in Daoy medulloblastoma cells. (A) mRNA from Daoy cells overexpressing $P A X 2 \mathrm{~b}$ and cotransfected with $d 2$ PAX2 specific or scrambled (scr) siRNA was investigated for $P A X 2$ expression. (B) Specifity was verified by determination of mRNA expression of GDNF, a known target gene of $P A X 2$. (C) Inversely, GDNF is upregulated in Daoy cells overexpressing $P A X 2 b$ compared to Daoy cells transfected with control vector. (D) $P A X 2$ downregulation by $d 1$ and $d 2 P A X 2$ specific siRNAs compared to $s c r$ siRNA in A172 glioma cells A172 and EJ bladder carcinoma cells shown by Western blotting. See top lane for $P A X 2$ at $47 \mathrm{kDa}$ and bottom lane for actin at $45 \mathrm{kDa}$.

glioma cell lines as well as the immortalized astrocytic cell line SV-FHAS (Fig. 3).

GDNF is regulated by PAX2 in Daoy medulloblastoma cells. Transient transfection of Daoy and T98G cells with two distinct $P A X 2$ targeted siRNAs $d 1$ and $d 2$ compared to scrambled (scr) control siRNA, transfection medium or growth medium alone was performed. The specificity of the siRNAs was demonstrated by co-suppression of $P A X 2$ as well as the $P A X 2$ target gene GDNF in Daoy medulloblastoma cells stably overexpressing
$P A X 2 \mathrm{~b}$ (Fig. 4A and B). Inversely, GDNF was upregulated in Daoy cells overexpressing $P A X 2 \mathrm{~b}$ compared to neo control cells (Fig. 4C). This fact is essential as it demonstrates both the dependency of GDNF on PAX2 expression as well as the functionality of the $P A X 2$ vectors used in our study. GFAP, nestin, NSE and vimentin which are not known to be $P A X 2$ targets were not regulated (data not shown). Since rapid cell death was occurring following $P A X 2$ downregulation in Daoy cells (see below), we were not able to show the effect on PAX2 protein level in this cell line. Instead we employed 

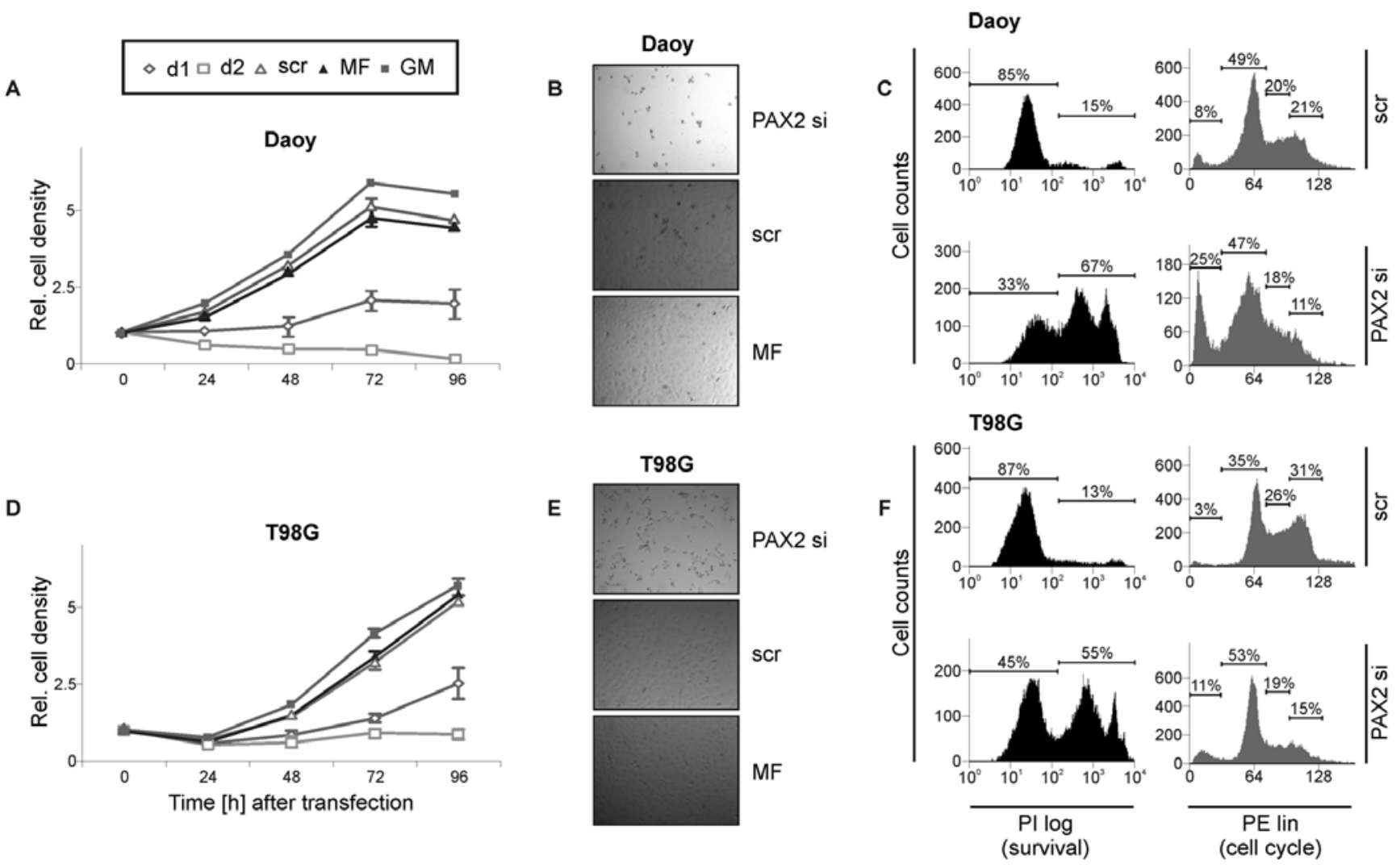

Figure 5. Gene suppression of $P A X 2$ induces cytotoxic effects in Daoy and T98G cells. Effect of $P A X 2$ specific siRNAs $d 1$ and $d 2$ compared to scrambled (scr) siRNA, Metafectene Pro transfection reagent (MF) or growth medium alone (GM) on Daoy (A) and T98G (D) cells as shown by crystal violet assay. Lightmicroscopic aspect of Daoy (B) and T98G (E) cells transfected with PAX2 specific siRNA molecules (d2) compared to scr siRNA (scr) or Metafectene Pro transfection reagent (MF) $24 \mathrm{~h}$ after transfection. Effects of transfection with $d 2$ or $s c r$ siRNA on cell viability and DNA content measured by PI staining and flow cytometry in Daoy (C) and T98G cells (F) $24 \mathrm{~h}$ after transfection. Cell death and cell cycle assays are shown on the right and far right side, respectively. High PI uptake (PI log) indicates cell death with disruption of the cell membrane (right side). Percentages of cell cycle analysis quantify sub G1/G0 peaks of apoptotic cells, G0/G1 phase, $\mathrm{S}$ phase and G2/M phase (far right side).

the glioma cell line A172, where the effect on cell survival was less pronounced and the bladder carcinoma cell line EJ to show the effect on PAX2 protein level. Efficient gene suppression in these two cell lines was demonstrated by Western blot analysis (Fig. 4D).

Gene suppression of PAX2 induces cytotoxic effects in Daoy and T98G cells. Transient transfection of Daoy cells with two distinct PAX2 targeted siRNAs $d 1$ and $d 2$ had profound cytotoxic effects as shown by crystal violet assay and phase contrast photography compared to $s c r$ control siRNA, transfection medium or growth medium alone (Fig. 5A and B). Flow cytometric analysis with PI-staining demonstrated an elevated rate of cell death occurring in cells treated with $P A X 2$ specific siRNA. Cell cycle analysis employing flow cytometry showed an elevated sub-G1 peak indicating apoptosis as well as a decreased number of cells in S- and G2/M-phase (Fig. 5C). A similar effect was observed in T98G malignant human glioma cells (Fig. 5D-F). However, overexpression of Pax2 in glioma cell lines did not enhance proliferation, suppress apoptosis or alter growth in the syngeneic VMdK mouse model (data not shown).

PAX2 expression in glioma: in silico analyses and correlation with markers of neuronal lineage differentiation. In glioma cell lines expression of $P A X 2$ was observed in only a fraction. To elucidate possible mechanisms of $P A X 2$ expression in gliomas, we followed a candidate gene approach and performed in silico analyses of glioma mRNA profiling datasets. With the candidate gene approach, we tested the hypothesis that $P A X 2$ was part of a neuronal signature. Expression of markers known to be involved in neuronal differentiation were investigated in the panel of glioma cell lines, Daoy cells and SV-FHAS cells by qRT-PCR. The data are summarised in Fig. 3. While several of the neuronal markers were highly expressed in glioma congruent with the increasing awareness of neuronal features in glioma (20), no correlation of $P A X 2$ with these markers was apparent. We also established immunohistochemistry on cell pellets of Daoy medulloblastoma and the twelve glioma cell lines as well as the stable PAX2 transfectants used in our study to assess GFAP, MAP2, NeuN, vimentin and synaptophysin protein expression. However, we observed no difference between $P A X 2$-positive and -negative cell lines or between $P A X 2$ overexpressing cell lines and their controls (data not shown). In silico analyses were performed with the Oncomine registry. According to the Pomeroy gene expression analysis (21), PAX2 was upregulated 1.889 -fold in classic and 1.894-fold in desmoplastic medulloblastoma samples compared to normal cerebellum probes. GDNF as a $P A X 2$ target gene was upregulated 2.569 -fold in classic 
A
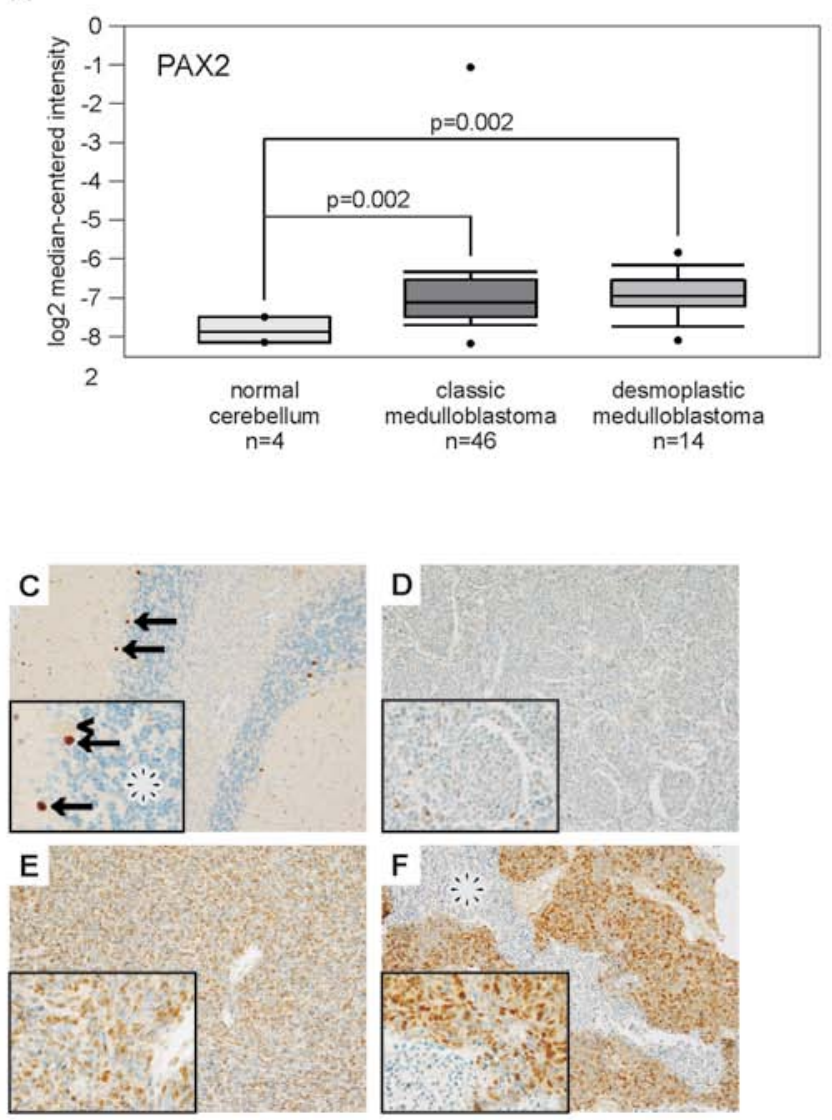
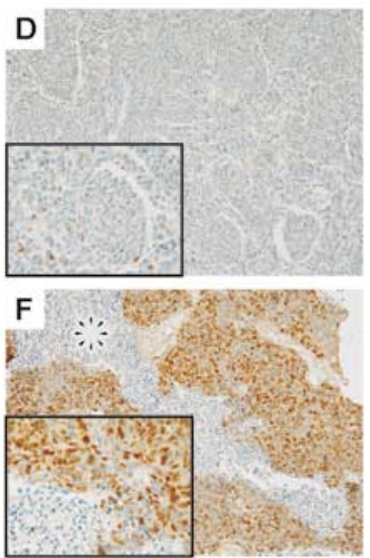

B

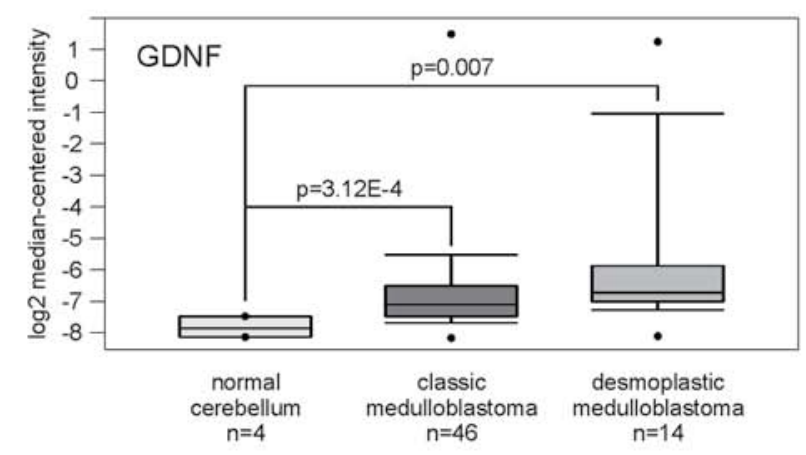

G

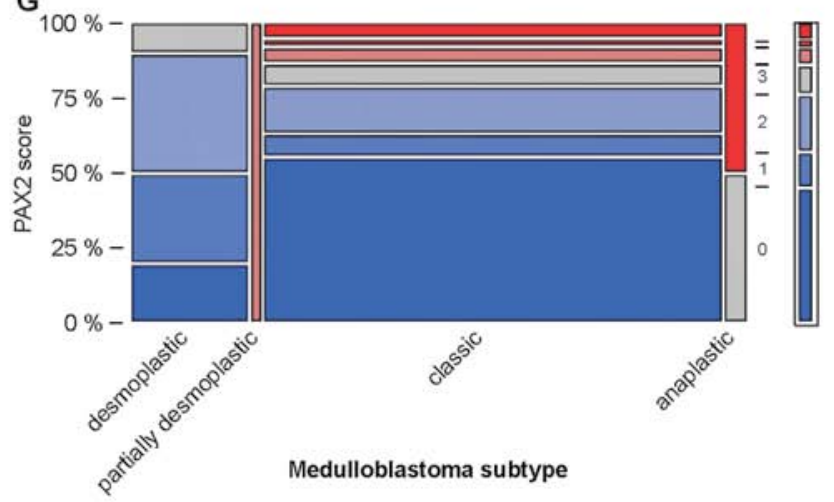

Figure 6. PAX2 expression increases with the degree of dedifferentiation in medulloblastoma. Elevation of $P A X 2$ expression by the factor of $1.889 / 1.894$ (A) and GDNF as a $P A X 2$ target gene by the factor of 2.569/3.97 (B) in classic/desmoplastic medulloblastoma samples compared to normal cerebellum probes according to Oncomine registry (Pomeroy brain dataset). Expression levels are normalised to the $\log 2$ median-centered intensity of the gene expression arrray. Boxed areas display the 75 th percentile, the median and the 25 th percentile. Error bars delineate the 90 th and 10th percentiles while the dots specify the maximum and minimum values. PAX2 immunohistochemistry of the normal cerebellum (C), basket cells (arrows), Purkinje cells (inset, arrowhead) or granular cells (inset, asterisk). Exemplary, PAX2 immunohistochemistries of desmoplastic subtype (D), classic medulloblastoma subtype (E) and anaplastic medulloblastomas are shown (asterisk indicating tumor necrosis) (F). Statistical analysis of nuclear PAX2 expression according to the medulloblastoma subtype using contingency analysis showing the Multi-score proportions of PAX2 expression in medulloblastoma specimens (G). The percentage fractions of each Multi-score according to medulloblastoma variants were analysed by a contingency analysis $\left(\mathrm{P}<0.005\right.$ in Pearson's $\chi^{2}$ test). The area of each box corresponds to the amount of specimens falling in the equivalent specific group of Multi-score analysis of $P A X 2$ expression.

and 3.97-fold in desmoplastic medulloblastoma samples (Fig. $6 \mathrm{~A}$ and $\mathrm{B})$. There was no correlation between PAX2 expression in medulloblastoma and the subtype-specific markers SFRP1, KCNA1, CTNNB1 or NPR3 (22). Also no correlation between $P A X 2$ expression and the negative predictors MYC, MYCN or CDK6 in medulloblastoma was apparent. In glioma, no significant correlation with neuronal markers defining subclasses of high-grade glioma (20) was found. Moreover, $P A X 2$ was not inversely correlated with p53 mRNA levels (data not shown).

PAX2 expression is restricted to basket cells in the normal cerebellum and increases with the degree of dedifferentiation in medulloblastomas. Because of the biologic function of $P A X 2$ in hindbrain development and the published data on deregulated $P A X 5$ expression in medulloblastomas as well as our RNA interference data in Daoy cells strongly suggesting a role for $P A X 2$ in medulloblastomas, we investigated PAX2 protein levels in medulloblastoma samples from two separate tissue microarrays. We found that in normal cerebellum,
PAX2 expression was largely absent. However, basket cells commonly had nuclear $P A X 2$ expression (Fig. 6C). In medulloblastomas, nuclear $P A X 2$ expression significantly increased with a less differentiated histomorphological phenotype: while virtually all desmoplastic/nodular medulloblastomas were $P A X 2$-negative (Fig. 6D) and intermediate nuclear $P A X 2$ levels were observed in the classic subtype (Fig. 6E), the anaplastic subtype exhibited strong nuclear $P A X 2$ expression (Fig. 6F). The statistical analysis of $P A X 2$ expression related to the medulloblastoma subtypes revealed significant differences $\left(\mathrm{P}<0.005\right.$ in Pearsons $\chi^{2}$ test) showing highest $P A X 2$ levels in medulloblastomas with the lowest histological degree of differentiation and lowest levels in medulloblastomas with higher differentiated morphology (Fig. 6G).

\section{Discussion}

Our study demonstrates that $P A X 2$ is aberrantly expressed and has antiapoptotic properties in medulloblastoma. Regarding the in vitro work, we found PAX2 to be overexpressed in the 
model medulloblastoma cell line Daoy (Fig. 1). Importantly, by transiently transfecting Daoy medulloblastoma cells with $P A X 2$ specific siRNA molecules we were able to induce a high level of cell death (Fig. 5). The cytotoxic effect of downregulating $P A X 2$ gene expression is in agreement with other studies demonstrating a potent antiapoptotic function of PAX proteins in melanoma, ovarian, bladder and prostate carcinoma cell lines $(13,23,24)$. In silico analysis of the Oncomine registry confirmed that $P A X 2$ expression is elevated in medulloblastoma compared to normal cerebellum (Fig. 6A and B). However, no correlation of $P A X 2$ expression with subtype-specific markers was found. In gliomas, neither our analysis of candidate genes (Fig. 3) nor in silico analyses were able to identify a neuronal signature correlating with $P A X 2$ expression. Thus, no obvious role of expression of $P A X$ genes in the evolution of the neuronal phenotype in gliomas was observed. Finally, our analysis of the expression of PAX2 protein in two tissue microarrays (TMA) containing 61 medulloblastoma samples not only confirmed aberrant $P A X 2$ expression in medulloblastoma samples on the protein level but also demonstrated a correlation with a less well differentiated phenotype. Of note this analysis also identified previously unknown $P A X 2$ expression in normal cerebellum with restriction to basket cells (Fig 6C-G).

In conclusion, $P A X 2$ represents a biomarker of more aggressive medulloblastoma and may have potential as a novel therapeutic target. The prognostic and predictive value of $P A X 2$ expression should be further validated in prospective studies of medulloblastoma.

\section{Acknowledgements}

The Dr Senckenberg Institute of Neurooncology is supported by the Hertie foundation and the Dr Senckenberg foundation. J.S. is 'Hertie Professor for Neurooncology'. This work was supported by the graduate college 686-1 'Mechanismen der Entstehung solider Tumoren und experimentelle Therapie-Konzepte'. We thank Professor Meinrad Busslinger and Professor Magdalena Götz for the productive discussions and professional advice.

\section{References}

1. Maulbecker CC and Gruss P: The oncogenic potential of Pax genes. EMBO J 12: 2361-2367, 1993.

2. Stuart ET and Gruss P: PAX: developmental control genes in cell growth and differentiation. Cell Growth Differ 7: 405-412, 1996.

3. Urbanek P, Fetka I, Meisler MH and Busslinger M: Cooperation of Pax 2 and Pax 5 in midbrain and cerebellum development. Proc Natl Acad Sci USA 94: 5703-5708, 1997.

4. Dressler GR, Wilkinson JE, Rothenpieler UW, Patterson LT, Williams-Simons L and Westphal H: Deregulation of Pax-2 expression in transgenic mice generates severe kidney abnormalities. Nature 362: 65-67, 1993.

5. Galili N, Davis RJ, Fredericks WJ, et al: Fusion of a fork head domain gene to PAX3 in the solid tumour alveolar rhabdomyosarcoma. Nat Genet 5: 230-235, 1993.

6. Busslinger M, Klix N, Pfeffer P, Graninger PG and Kozmik Z: Deregulation of PAX-5 by translocation of the Emu enhancer of the IgH locus adjacent to two alternative PAX-5 promoters in a diffuse large-cell lymphoma. Proc Natl Acad Sci USA 93: 6129-6134, 1996.
7. Kroll TG, Sarraf P, Pecciarini L, et al: PAX8-PPARgamma1 fusion oncogene in human thyroid carcinoma. Science 289: 1357-1360, 2000 .

8. Murer L, Caridi G, Della Vella M, et al: Expression of nuclear transcription factor PAX2 in renal biopsies of juvenile nephronophthisis. Nephron 91: 588-593, 2002.

9. Winyard PJ, Risdon RA, Sams VR, Dressler GR and Woolf AS: The PAX2 tanscription factor is expressed in cystic and hyperproliferative dysplastic epithelia in human kidney malformations. J Clin Invest 98: 451-459, 1996.

10. Gnarra JR and Dressler GR: Expression of Pax-2 in human renal cell carcinoma and growth inhibition by antisense oligonucleotides. Cancer Res 55: 4092-4098, 1995.

11. Hueber PA, Waters P, Clark P, Eccles M and Goodyer P: PAX2 inactivation enhances cisplatin-induced apoptosis in renal carcinoma cells. Kidney Int 69: 1139-1145, 2006.

12. Torban E, Eccles MR, Favor J and Goodyer PR: PAX2 suppresses apoptosis in renal collecting duct cells. Am J Pathol 157: 833-842, 2000.

13. Muratovska A, Zhou C, He S, Goodyer P and Eccles MR: Paired-Box genes are frequently expressed in cancer and often required for cancer cell survival. Oncogene 22: 7989-7997, 2003.

14. Stuart ET, Haffner R, Oren M and Gruss P: Loss of p53 function through PAX-mediated transcriptional repression. EMBO J 14: 5638-5645, 1995.

15. Steinbach JP, Klumpp A, Wolburg H and Weller M: Inhibition of epidermal growth factor receptor signaling protects human malignant glioma cells from hypoxia-induced cell death. Cancer Res 64: 1575-1578, 2004

16. Livak KJ and Schmittgen TD: Analysis of relative gene expression data using real-time quantitative PCR and the 2(-Delta Delta C(T)) method. Methods 25: 402-408, 2001.

17. Mittelbronn M, Capper D, Bunz B, et al: De novo erythropoietin receptor (EPO-R) expression in human neoplastic glial cells decreases with grade of malignancy but is favourably associated with patient survival. Neuropathol Appl Neurobiol 33: 299-307, 2007.

18. Louis DN, Ohgaki H, Wiestler OD, et al: The 2007 WHO classification of tumours of the central nervous system. Acta Neuropathol 114: 97-109, 2007.

19. Liao CL, Lee MY, Tyan YS, et al: Progesterone receptor does not improve the performance and test effectiveness of the conventional 3-marker panel, consisting of estrogen receptor, vimentin and carcinoembryonic antigen in distinguishing between primary endocervical and endometrial adenocarcinomas in a tissue microarray extension study. J Transl Med 7: 37, 2009.

20. Phillips HS, Kharbanda S, Chen R, et al: Molecular subclasses of high-grade glioma predict prognosis, delineate a pattern of disease progression, and resemble stages in neurogenesis. Cancer Cell 9: $157-173,2006$.

21. Pomeroy SL, Tamayo P, Gaasenbeek M, et al: Prediction of central nervous system embryonal tumour outcome based on gene expression. Nature 415: 436-442, 2002.

22. Remke M, Hielscher T, Northcott PA, et al: Adult medulloblastoma comprises three major molecular variants. J Clin Oncol 29: 2717-2723, 2011.

23. Scholl FA, Kamarashev J, Murmann OV, Geertsen R, Dummer R and Schafer BW: PAX3 is expressed in human melanomas and contributes to tumor cell survival. Cancer Res 61: 823-826, 2001.

24. Gibson W, Green A, Bullard RS, Eaddy AC and Donald CD: Inhibition of PAX2 expression results in alternate cell death pathways in prostate cancer cells differing in p53 status. Cancer Lett 248: 251-261, 2007.

25. Ishii N, Maier D, Merlo A, et al: Frequent co-alterations of TP53, p16/CDKN2A, p14ARF, PTEN tumor suppressor genes in human glioma cell lines. Brain Pathol 9: 469-479, 1999. 